\title{
Biology of and Implications for the Treatment of Malignant Pleural Mesothelioma
}

\author{
Harvey I. Pass MD
}

Pleural mesothelioma (MPM) is a neoplasm for which standard treatment regimens, including surgery, chemotherapy, and radiation, have had no impact on median survival (approximately 8-10 months from diagnosis). Although relatively rare, it is estimated that there will be over 80,000 new cases of the disease over the next 20 years. As with other thoracic malignancies, it would certainly be to the clinicians advantage to know how these malignancies develop and propagate in order to be able to precisely formulate treatment strategies. Paralleling the rising incidence of mesothelioma, there has been an acceleration of interest in fiber carcinogenesis, autocrine growth pathways, molecular genetics, and altered gene products in mesothelioma.

The generation of mesothelioma cell lines has enabled researchers to perform karyotyping analysis to detect specific chromosomal deletions, translocations, and inversion which could give hints regarding the loss of genetic material. Such losses could downregulate or completely abolish the action of tumor suppressor genes, leading to uninhibited growth. The development of enzyme-linked assays allow quantitation of abnormal secretory products from the cells lines which may represent autocrine growth factórs. Molecular biologic techniques including Northern (RNA) and Southern blot analysis (DNA), as well as the polymerase chain reaction (which amplifies DNA segments from only a few oligonucleotides) are documenting specific results of chromosomal damage, including base sequence changes. In vitro/vivo chemo and radiation sensitivity studies have been applied to cell lines and animal models to add preclinical justification for new treatment protocols.

This review details some of the newer developments in the cellular and molecular biology of pleural mesothelioma. By definition, it is an incomplete review due to space constraints of the manuscript. Nevertheless, the emphasis will be on the overall picture of mesothelioma carcinogenesis. The most important aspect of this discussion is that a multitude of known and unknown factors must be combined in order to transform and propagate mesothelial cells. Fu-

Professor of Surgery and Oncology Wayne State University

Chief of Thoracic Oncology Aerodigestive Program Director Karmanos Cancer Institute Detroit, Michigan 
ture therapies may be geared toward the resolution of these molecular abnormalities, either by substituting the "correct" genetic material causing differentiation to the normal phenotype, or genetically interfering with the factors necessary for the tumor to divide.

Until these disease markers are identified, and novel solutions formulated, clinicians will use therapies which are a combination of standard techniques or utilizing newer technologies which are not specifically targeted toward the genetic material. These protocols may be multimodal in their approach, combining surgery with newer methods of treatment delivery, or they may involve completely new approaches.

\section{Asbestos Effects}

There are two families of asbestos fibers: curly and pliable serpentine (Chrysotile), and the rod-like amphiboles which include Crocidolite, Amosite, and Anthrophylite, Tremolite, and Actinolyte ${ }^{1)}$. Long $(>4 \mu \mathrm{M})$, thin $(<0.25 \mu \mathrm{M}$ diameter $)$ are more carcinogenic than short, thick fibers. Asbestos fibers $^{2)}$, may promote or initiate carcinogenesis through a variety of mechanisms, and crucial to this development are individual characteristics of the fibers themselves ${ }^{3)}$. Inhaled fibers are cleared from the tracheobronchial tree by macrophages and ciliary action, and the remaining fibers accumulate in the lower third of the lungs adjacent to the visceral pleura ${ }^{2)}$. As opposed to crocidolite and other amphiboles, the serpentine fibers break down into smaller subunits which deteriorate or dissolve in the lung and are then eliminated by lymphatics.

Asbestos fibers promote mesothelioma carcinogenesis through a number of different mechanisms including free radical effects, effects on immune function, as well a by direct physical effects on chromosomes.

\section{Free Radical Effects}

Asbestos fibers can produce reactive oxygen species, the most toxic of which are hydroxyl radical and superoxide, and provide an important source of metal cations (iron) which promote the generation of hydroxyl radicals from hydrogen peroxide, with resulting DNA damage ${ }^{4}$. These oxygen radicals can promote carcinogenesis through direct DNA interactions, membrane (lipid) peroxidation, activation of oncogenes, and disruption of cellular defenses.

Asbestos can also interact with monocytes, macrophages, and neutrophils to cause free radical generation through phagocytosis-induced augmentation of the respiratory burst. Our laboratory has been most interested in these secondary asbestos effects from two perspectives : whether asbestos can induce production of nitric oxide by effector cells or cause mononuclear cells to release inflammatory cytokines such as TNF or the interleukins. Nitric oxide is a bioregulatory agent which has many important physiologic functions including macrophage mediated tumor cytotoxicity and antimicrobial activity. NO can accelerate base deamination with base transitions and mutations in mammalian DNA ${ }^{5}$. Moreover, macrophage nitric oxide has been found to have suppressive effects on T-cells, and to alter antigen presentation, which may impact on cancer surveillance ${ }^{6)}$. Prewitt and Pass have found that crocidolite asbestos fibers, in the presence of interferon, will induce the production of NO by a murine macrophage cell line. Treatment with fibers alone does not produce NO. This effect is selective for crocidolite fibers as opposed to 
the serpentines ${ }^{7)}$. The impplications of enhanced NO production are only speculative, but this effect may produce a local environment of immunosuppressive effects as stated above, thus allowing unimpeded carcinogenesis.

Prewitt and Pass have also noted that crocidolite was the only fiber to induce TNF $\alpha, \mathrm{IL}-1 \alpha, \mathrm{IL}-1 \beta$, and IL- 6 production by human monocytes. Neither amosite nor chrysotile were found to cause production of these inflammatory cytokines ${ }^{7)}$. These findings suggest that one aspect of the increased incidence of mesothelioma after exposure to crocidolite asbestos could be related to the selective inflammatory response of monocytes to these fibers.

Hence, asbestos fibers may induce cell transformation in part through nongenotoxic mechanisms in a multistage model of carcinogenesis $^{8}$. DNA damage mediated by either the physical location of perinuclear asbestos fibers or asbestos-generated free radicals may induce heritable genetic changes in the target cell that are irreversible but not sufficient to cause cell transformation. The elaboration of cytokines and growth factors by monocytic cells in response to fibers might then exert further genotypic changes and instigate cell proliferation that results in promotion and propagation of the initiated cell, resulting in the malignant phenotype.

\section{Asbestos and the Immune System}

Asbestos-exposed individuals and patients with mesothelioma have abnormal humoral and cell mediated immunity, cell mediated antibody-dependent cellular cytotoxicity, as well as defective function of macrophages and natural killer cells ${ }^{9) \sim 11}$. These individuals have elevated immunoglobulin levels and autoantibodies. All types of asbestos will significantly reduce lymphokine activated killer cell and natural killer cell function in vitro but will not affect the ability of the individual to generate lymphokine activated killer cells ${ }^{11)}$. Of significance, interleukin-2 cannot overcome the asbestosinduced suppression of natural killer or lymphokine activated killer activity. The capacity of asbestos fibers to induce chromosomal changes (as discussed below), as well as to suppress immune effector cell function, may result in a local environment of progressive non-reparative DNA damage.

\section{Chromosomal Effects}

Asbestos can cause transformation of rat and human mesothelial cells in vitro and alter their growth characteristics ${ }^{12,13)}$. The transformation of cells and accompanying chromosomal changes are frequently associated with aneuploidy. The mechanism for the aneuploidy could be related to interference with chromosomal segregation, resulting in anaphase abnormalities ${ }^{14), 15}$. The asbestos fibers themselves most likely physically interfere with the spindle apparatus resulting in the aneuploidy. The ability of the cells to phagocytize longer, thinner, fibers leads to greater accumulation of the asbestos with a greater chance for chromosomal migratory abnormalities ${ }^{16}$. The resulting deletions and translocations have been described in a number of mesothelioma cell lines, specifically involving chromosomes $1,2,3,4,5,6,7,9,11$, 17 and 22 (See later). As will be discussed in a later section, alteration of growth factor, specifically PDGF and/or IGF, may contribute to autocrine or paracrine stimulation of mesothelioma cell growth, or chromosome damage with gene deletions or point 
mutations may cause oncogene amplification.

In a group of nine newly developed cell lines from mesothelioma patients participating in trials at the NCI, aneuploidy with chromosome numbers between 40 and 88 have been noted. Karyotypic analysis of these lines has revealed deletions, inversions, or translocations most frequently in chromosome 1 (100\%), 3 (67\%), and 9 (56\%) (Pass et al., in press). These data have recently been confirmed by others. The following discussion will concentrate on the more common nonrandom aberrations seen in mesothelioma karyotypes.

The changes in chromosome 1 are partial loss due to deletion or rearrangement frequently involving bands $1 \mathrm{p} 11-\mathrm{pter}^{17) \sim 19)}$. The shortest region of overlap for these deletions occurs at $1 \mathrm{p} 21-\mathrm{p} 22$ in the samples described by Flejter et al, and it is theorized that may be the site of a putative tumor suppressor gene important in mesothelial cell tumorigenesis ${ }^{17)}$.

Chromosome 3 changes can be seen in up to $90 \%$ of cases studied and involve deletions and rearrangement in the involved region $\mathrm{p} 14^{-} \mathrm{p} 25^{17), 19)}$. The shortest region of overlap is thought to be at $3 \mathrm{p} 21^{17), 18)}$. Zeiger and Pass examined the 9 previously mentioned new NCI mesothelioma cell lines, their corresponding solid tumors, and 15 additional mesothelioma solid tumors for loss of heterozygosity in the region from $3 \mathrm{p} 13-3 \mathrm{p} 24$ using the PCR-based RFLP analysis at 7 loci. This term, loss of heterozygosity (LOH), asks whether one of the two alleles at a particular chromosomal site has been lost during the development of a tumor. This is done by comparing regions of interest in the tumor DNA with DNA from the patients normal tissue. The loss of an allele could imply the presence of a recessive gene important in the development of the tumor. Overall, $4 / 9$ or $44 \%$ of the mesothelioma cell lines and 6 of the 15 solid tumors (40\%) demonstrated LOH at loci on 3p. The documentation of this reproducible loss of genetic material on chromosome 3 suggests the possibility for loss of a tumor suppressor gene $e^{20), 21)}$.

Monosomy 4 has been observed as a nonrandom event in hypodiploid and hypotetraploid karyotypes ${ }^{18), 22}$.

Chromosome 6 has been the site of many structural rearrangements that can lead to deletion of the long arm, particularly at bands q15-q24 ${ }^{23)}$.

Polysomy 7 (partial or complete), especially of the $\mathrm{p}$ arm has been associated with mesothelioma ${ }^{21), 23) \sim 25)}$. The copy number of chromosome $7 \mathrm{p}$ arm was found to be inversely correlated with survival ${ }^{21 \text { ) }}$.

Loss of chromosome $9 \mathrm{p}$ has been noted to be a consistent feature of hypodiploid and hypotetraploid karyotypes ${ }^{18), 19), 21)}$. In a recent series $80 \%$ of cases revealed homozygous or hemizygous deletions on $9 p$ with the shortest region of overlap being 9p21-22. In a collaboration with Dr. Bruce Johnson of the NMOB, Pass will attempt to corroborate these data in solid tumor to make sure than this interesting loss in the region of the interferon locus is not a culture artifact. This loss has also been seen with leukemia, glioma, melanoma, and lung and bladder cancers. These deletions are in the vicinity of the interferon loci. Homozygous (complete) losses of $9 p$ in this location were identified in $43 \%$ of cases and may represent the inactivation of a tumor suppressor gene in this location.

Rearrangements of chromosome 11 and a common breakpoint at $11 \mathrm{q} 13$ with translocation have been observed ${ }^{26}$. 
Monosomy 22 (partial or total) has been a common findings in many studies ${ }^{21), 24), 25)}$. Partial loss or translocation involving the $\mathrm{q}$ arm with common breakpoint at $22 \mathrm{q} 11$ is seen.

Since common non-random cytogenetic abnormalities have been found in mesothelioma, the next area of interest would be to identify the genes affected by the chromosomal rearrangements. Theses can be generally categorized as tumor suppressor genes or prote/oncogenes

\section{Tumor Suppressor Genes and Proto/ Oncogene Changes}

The location of protooncogenes/tumor suppressor genes have been mapped to specific chromosome regions, e. g. N-ras in band 1p13, EGF-r in band 7p12-p13, PDGF $\beta$ in band $22 \mathrm{q} 12.3^{-\mathrm{q} 13.1}$, etc. The presence of extra copies of chromosoms may represent a mechanism for protooncogene amplification whereas loss of a chromosome may cause a loss of a tumor suppressor gene. Translocation and subsequent gene rearrangement may allow activation of a protooncogene or deactivation of a tumor suppressor gene. Although there is little comprehensive information available, there has not been consistent evidence of gene rearrangement or amplification for the following oncogenes or tumor suppressor genes in the pathogenesis of MPM : EGF-r, myc, Her 2/ neu, rb, and ras. Recently, however, there has been a great deal of interest in p53 as well as new data on Wilms tumor suppressor gene status in MPM.

p53

Alterations in chromosome 17, specifically $17 \mathrm{p}$, have been described in a variety of tumors, including mesothelioma. This encodes for the p53 protein which is believed to be the product of a tumor suppressor gene ${ }^{27) ~ 32)}$. Mutations of this gene (which is normally expressed as "wild type") have been reported to be frequent events in a variety of tumor types, resulting in a defect in the activation of tumor suppressor activity. Ironically, growth promoting activity of mutant p53 has been described. The mutant protein is actually more stable than the wild type and will accumulate in the anaplastic cells. Specific antibodies to the mutant protein can be used for immunohistochemical analysis of paraffin fixed or snap frozen specimens. The presence of the mutant protein implies that there has been a mutation which has increased its half-life.

Cell lines derived from murine models of asbestos-induced mesothelioma have reduced or absent expression of p53 messenger RNA compared to the RNA from nontumorigenic cell lines or reactive mesotheial cells ${ }^{32)}$. In human cell lines derived from patients with malignant mesothelioma, attention has turned to specific mutations or genetic abnormalities in p53. Abnormalities have been reported in chromosome 17 which is the location of the p53 gene. Cote, in a study of four human mesothelioma cell lines found two of the four tumors had cytogenetic abnormalities of chromosome 17 , and that two of the tumors had loss of heterozygosity in the $17 \mathrm{p} 13$ region $^{31}$. Sequence analysis revealed single base substitutions in two of the tumor cell lines. This group expanded their investigations to 20 cell lines of which 10 had abnormalities of chromosome 17p. Seven of the 20 lines (35\%) had immunohistochemical increased expression of $\mathrm{p} 53^{33)}$. These data are in sharp contrast to that of Metcalf who found p53 abnormalities in only 3 of twenty mesothelioma cell lines of which 2 were point 
mutations $^{30}$. In an analysis of 32 mesotheliomas, Mew and Pass found evidence for base pair mutations in the evolutionarily conserved exons 5-9 in only 3 cases. Moreover, of 15 evaluable cases, we have observed loss of heterozygosity in only 2 . These data, then, seem to confirm only minor role for $\mathrm{p} 53$ in the pathogenesis of mesothelioma. Wilms Tumor Gene

A putative tumor suppressor gene WT1 linked to chromosome 11 has been implicated in the pathogenesis of Wilms tumor. When there is a mutation inactivating one germline allele of WT1, there is a genetic predisposition to Wilms tumor, and about $10 \%$ of sporadic Wilms tumor specimens contain a mutation of WT1. WT is only expressed in certain tissues, but is expressed in adults in connective structures of mesothelial origin. $\mathrm{Park}^{34)}$ has recently described a patient with non-asbestos related mesothelioma whose WT1 was inactivated by a point mutation, leading to the hypothesis that inactivation of this tumor suppressor gene could lead to the production of transformed mesothelial tissue. Unfortunately, the screening of 32 specimens of asbestos-related mesothelioma did not reveal WT1 mutations. As stated above, however, a number of cases show chromosome 11 arrangements in cell lines from asbestos related mesothelioma. Northern Blot analysis of RNA from cell lines derived from NCI patients has revealed the presence of WT1 transcripts, as opposed to the lack of transcripts in the RNA from nonsmall cell lung cancers (Amin, Albelda, Kaiser, Pass, data unpublished). Moreover, the same pattern has been seen in animal models of spontaneous and asbestos induced rat mesothelioma (Walker and Pass, Cancer Research, in press). Such specificity could be of use in the differential diagnosis of the disease.

\section{Platelet Derived Growth Factor}

One of the more intriguing oncogenes in malignant mesothelioma is $\mathrm{C}$-sis, which codes for one of the two chains ( $\alpha$ and $\beta$ ) of platelet derived growth factor (PDGF) ${ }^{35}$. The relationship between $\mathrm{PDGF}-\beta$ and transformation was first realized when the peptide chain was found to be homologous to the viral cis oncogene. When this cis oncogene is transfected into fibroblasts they become transformed into a malignant phenotype ${ }^{36)}$. Because of the closeness of this oncogene with the PDGF- $\beta$ chain it is felt that PDGF$\beta$ may work through an autocrine regulatory system for cell growth. The relative contribution of the $\alpha$ and $\beta$ chains in the transformation process in controversial. Nevertheless, the confirmation of specific receptors for the PDGF molecules occurring within the cell and on the cell surface adds credence to an autocrine stimulatory loop for mesothelioma via this pathway ${ }^{37}$. Gerwin was the first to describe elevation of RNA levels for both the $\alpha$ and $\beta$ chains of PDGF in mesothelioma cell lines and correlated the increase with PDGF-like activity secreted by the cells ${ }^{38}$. Pogrebniak and Pass have confirmed progressive increases in PDGF $\mathrm{AB}$ in the conditioned media of growing mesothelioma cell lines. These data were corroborated by Versnel who described elevation of both PDGF chains in malignant mesothelioma cell cultures compared to normal cells, but the elevation was chiefly in the $\beta$ chain $^{37)}$. Pass and Pogrebniak investigated the expression of $\mathrm{PDGF}-\beta$ mRNA in tumor specimens excised from 16 patients with MPM. Northern blot analyses revealed elevation of PDGF- $\beta$ expression above control (PDGF- $\beta$ mRNA expression 
from freshly elutriated human peripheral blood monocytes stimulated with transforming growth factor- $\beta$ TGF) in $10 / 16$ (63\%) of MPM patients. A $230 \%$ increase in PDGF- $\beta$ expression was found between the lowest and highest expression samples. The specimens from which the PDGF transcripts were derived were found histologically to contain $87 \%$ tumor and $13 \%$ contaminating normal cells, predominantly lymphocytes ${ }^{39}$.

Of interest is the frequent association of abnormalities of chromosome 22 in malignant mesothelioma which is the site of the $\mathrm{C}$-sis gene : yet, to date, no specific rearrangements of the $\mathrm{C}$-sis gene have been described to explain its altered activity. A possible autocrine growth loop in which the PDGF secreted by the tumor could cause self stimulation has been hypothesized since malignant mesothelioma cells have been found to possess specific receptors for PDGF- $\beta$ while lacking the expression of $\alpha$ receptors ${ }^{37)}$. This is in marked contrast to normal mesothelioma cells which have $\alpha$ receptors but little or no $\beta$ receptors. If, indeed, PDGF is involved in autocrine stimulation of mesothelioma, future strategies for treatment could involve receptor targeting.

Other Growth Factors and Cytokines

Lee reported that normal human mesothelial lines as well as human mesotheliomas express IGF-1, and IGF-1 receptor by Northern blot or by PCR. IGF-1 acts synergistically with PDGF to promote mesenchymal proliferation ${ }^{40}$. Pass has noted that a hamster model of mesothelioma induced by SV40 viral infection (see later) is associated with $\mathrm{IGF}-1$ receptor expression as well as production of IGF-1. Moreover, in human lines as well as frozen tissue from resected mesothelioma patients, the majority will express IGF-1 receptor by immunoprecipitation (Procopio and Pass, in process).

Besides growth factors such as PDGF and IGF, there may be a role for cytokines in the pathogenesis of mesothelioma. The association between thrombocytosis and mesothelioma has stimulated investigations looking for increased circulating levels of cytokines such as interleukin-3, interleukin4 , granulocyte macrophage colony stimulating factor, erythropoietin, stem cell factor, interleukin-11 and interleukin-6. Pass has substantiated reports of high levels of interleukin- $6^{41,42)}$ and granulocyte macrophage colony stimulating factor ${ }^{43)}$ from body fluids or cell lines of patients with mesothelioma. Concurrently, Pass not seen altered expression in the media of epidermal growth factor or $\mathrm{TGF}-\alpha$ or $\beta$. Other investigators postulate other growth factors apart from EGF, PDGF, and TGF-beta, as yet uncharacterized, may play a role in autocrine or paracrine growth loops in this disease $\mathrm{e}^{44)}$.

\section{A Viral Connection?}

The issue of mesothelioma carcinogenesis is now further complicated by the finding that intrapleural injection of SVC40 virus will induce mesotheliomas in $100 \%$ of the animals, independent of asbestos exposure ${ }^{45)}$. In an analysis of 49 mesotheliomas having resection at our institution, $60 \%$ were found to have SV40 like DNA and the majority had expression of intranuclear $\mathrm{T}$ antigen, which is a well recognized consequence of SV40 infection. One wonders what the effect of incorporation of such virion DNA could have on growth promotion of mesothelial cells. This novel finding, however, may only be explained through intense epidemiologic examination of the patients exposure history. It is intriguing that asbestos is 
known to facilitate transformation of cells by SV40 DNA in vitro ${ }^{46)}$, and that SV40 incorporation can upregulate IGF-1 receptor status. Such interrelationships among asbestos, viral particles, DNA damage with incorporation and subsequent upregulation of growth factor receptors may give clues to the ultimate details of MPM carcinogenesis. Moreover, in recent studies, Mew and Pass have shown that incorporation of antisense to TGF-1R DNA into hamster mesothelioma cell lines will halt growth of the cell lines compared to sense IGF-1R incorporation.

\section{Prospects for Future Therapies}

The ability to recognize specific genes involved in the carcinogenesis of mesothelioma, as well as growth factors which result from aberrant genetic material will have both diagnostic and therapeutic significance in the disease. Certain oncogene products may be recognizable as tumor markers, and be able to define some of the prognostic characteristics in a given patient. Neutralization of growth factors, either with antibody treatment or by interfering with receptor mediated uptake, can be investigated once those growth factors are defined. The introduction of foreign DNA for thera- peutic purposes (so called "gene-therapy") is being studied, and these approaches involve (1) introduction of sequences which inhibit the transcription and subsequent translation of factors which are important for growth of the cell (i. e. antisense technology) (2) introduction of sequences which will code for a given substance which, in the presence of exogenously added drugs, will cause cell death selectively to proliferating tissue (i. e. thymidine kinase insertion). Most recently, Mew and Pass have demonstrated that incorporation of the thymidine kinase gene into mesothelioma cells can as a suicide gene in the presence of gancyclovir. Moreover, as with other TK systems, a "bystander effect" at least in vitro seems to be operative. Thses findings are presently being expanded into animal models. Finally the introduction of DNA which has been lost through deletions (i. e. tumor suppressor genes), sot that there can be a dedifferentiation of the tumor to the normal phenotype. Refinement in the method of introduction of the foreign DNA, either by retroviral or adenoviral vectors, or by liposomal delivery, is crucial in order to deliver high enough levels of the genetic material so it can be efficiency incorporated.

\section{Bibliography}

1) Mossman B, Light W, Wei E : Asbestos : Mechanisms of toxicity and carcinogenicity in the respiratory tract. Ann Rev Pharmacol Toxicol $1983 ; 23: 595-615$.

2) Walker C, Everitt J, Barrett JC : Possible cellular and molecular mechanisms of asbestos carcinogenicity. Am J Ind Med $1992 ; 21$ : 253-273.

3) Stanton MF, Layard M, Tegeris A, et al : Relation of particle dimension to carcinogenicity in amphibole asbestoses and other fibrous minerals. J Natl Cancer Inst 1981 ; $67: 965-975$.

4) Zalma R, Bonneau L, Jaurand MC, Guignard $\mathrm{J}$, Pezerat $\mathrm{H}$ : Formation of oxy-radicals by oxygen reduction arising from the surface activity of asbestos. Can J Chem $1987 ; 65$ : 2338-2341.

5) Wink DA, Kasprzak KS, Maragos CM, et al : DNA deaminating ability and genotoxicity of nitric oxide and its progenitors. Science $1991 ; 254: 1001-1003$. 
6) Drapier J : L-arginine-derived nitric oxide and the cell-mediated immune response. Res Immunol $1991 ; 142: 553-602$.

7) Prewitt TW, Chaudhri G, Pass H : Asbestos and interferon gamma synergistically induce murine macrophages to produce nitric oxide. Proc AACR $1993 ; 34: 445$.

8) Cohen SM, Ellwein LB : Genetic errors, cell proliferation, and carcinogenesis. Cancer Res $1991 ; 51: 6493-6505$.

9) Hodson ME : Immune function and survival in pleural mesothelioma. Letter to the Editor. Clin Exp Immunol $1985 ; 61: 214-215$.

10) Lew F, Tsang P, Holland JF, Warner N, Selikoff IJ, Bekesi JG : High frequency of immune dysfunctions in asbestos workers and in patients with malignant mesothelioma. J Clin Immunol $1986 ; 6: 225-233$.

11) Manning LS, Davis MR, Robinson BWS : Asbestos fibers inhibit the in vitro activity of lymphokine-activated killer (LAK) cells from healthy individuals and patients with malignant mesothelioma. Clin Exp Immunol $1991 ; 84: 85-91$.

12) Jaurand $M:$ Observations on the carcinogenicity of asbestos fibers. Ann N Y Acad Sci $1992 ; 258-270$.

13) Rom WN, Travis WD, Brody AR : Cellular and molecular basis of the asbestos-related diseases. Am Rev Respir Dis 1991 ; 143 : 408422.

14) Hesterberg TW, Barrett JC : Induction by asbestos fibers of anaphase abnormalities :

Mechanism for aneuploidy induction and possibly carcinogenesis. Carcinogenesis $1985 ; 6: 473-475$.

15) Hesterberg TW, Brody AR, Oshimura M, Barrett JC: Asbestos and silica induce morphological transformation of mammalian cells in culture: A possible mechanism. In : Goldsmith DF, Winn DM, Shy CM, eds. Silica, Silicosis, and Cancer. New York : Praeger Press, 1986 : 177-190.

16) Hesterberg TW, Butterick CJ, Oshimura M, Brody AR, Barrett JC : Role of phagocytosis in Syrian hamster cell transformation and cytogenetic effects induced by asbestos and short and long glass fibers. Cancer Res 1986 ; $46: 5795-5802$.

17) Flejter WL, Li FP, Antman KH, Testa JR : Recurring loss involving chromosomes 1, 3, and 22 in malignant mesothelioma : Possible sites of tumor suppressor genes. Genes, Chromosomes, Canc $1989 ; 1: 148-154$.

18) Hagemeijer A, Versnel MA, van Drunen E, et al : Cytogenetic analysis of malignant mesothelioma. Cancer Gent Cytogenet 1990; $47: 1-28$.

19) Taguchi T, Zhou JY, Jhanwar SC, et al : Recurrent chromosome losses in human malignant mesothelioma. Proc Amer Assoc Cancer Res $1992 ; 33: 40$.

20) Roboz J, Chahinian AP, Holland JF, Silides D, Azrajer L : Early diagnosis and monitoring of transplanted human malignant mesothelioma by serum hyaluronic acid. J Natl Cancer Inst 1989 ; $81: 924-928$.

21) Tianinen M, Tammilehto L, Rautonen J, Tuomi T, Mattson K, Knuutila S : Chromosomal abnormalities and their correlations with asbestos exposure and survival in patients with mesothelioma. Br J Cancer 1989 ; $60: 618-626$.

22) Tammilehto L, Tuomi T, Tiainen M, et al : Malignant mesothelioma : clinical characteristics, asbestos mineralogy and chromosomal abnormalities of 41 patients. Eur J Cancer 1992; 28A : 1373-1379.

23) Tiainen M, Hopman A, Moesker O, et al : Interphase cytogenetics on paraffin sections of malignant pleural mesothelioma. Cancer Genet Cytogenet $1992 ; 62: 171-179$.

24) Tiainen M, Kere J, Tammilehto L, Mattson $\mathrm{K}$, Knuutila $\mathrm{S}$ : Abnormalities of chromosomes 7 and 22 in human malignant pleural mesothelioma : Correlation between southern blot and cytogenetic analyses. Genes, Chromosomes, Canc $1992 ; 4: 176-182$.

25) Tiainen M, Tammilehto L, Mattson K, Knuutila S : Nonrandom chromosomal abnormalities in malignant pleural mesothelioma. Cancer Genet Cytogenet 1988; 33 
: 251-274.

26) Gibas Z, Li FP, Antman KH, Bernal S, Stahel $\mathrm{R}$, Sandberg AA : Chromosome changes in malignant mesothelioma. Cancer Genet Cytogenet $1986 ; 20: 191-201$.

27) Kane MJ, Chahinian AP, Holland JF : Malignant mesothelioma in young adults. Cancer $6593 ; 1990: 1449-1455$.

28) Chang K, Ding IYF, Chang $X$, et al : Heterogeneous expression of p53 is a common feature of human malignant mesothelioma. Proc Amer Assoc Cancer Res $1992 ; 33: 382$.

29) Kafiri G, Thomas DM, Shepherd NA, Krausz $T$, Lane DP, Hall PA : p53 expression is common in malignant mesothelioma. Histopathology $1992 ; 21: 331-334$.

30) Metcalf RA, Welsh JA, Bennett WP, et al : p53 and Kirstein-ras mutations in human mesothelioma cell lines. Cancer Res 1992 ; $52: 2610-2615$.

31) Cote RJ, Jhanwar SC, Movick S, Pellicer A : Genetic alterations of the p53 gene are a feature of malignant mesotheliomas. Cancer Res $1991 ; 51: 5410-5416$.

32) Cora E, Kane A : Expression of the tumor suppressor gene, p53, during the development of murine malignant mesotheliomas induced by asbestos fibers. FASEB J $1991 ; 5$ : A1442.

33) Dlahi A, Gerdes H, Chen Q, Saad A, Jhanwar SC : Abnormalities of chromosome 17 and p53 locus in malignant mesothelioma. Proc Amer Assoc Cancer Res 1992 ; $33: 381$.

34) Park S, Schalling M, Bernard A, et al : The Wilms tumour gene WT1 is expressed in murine mesoderm-derived tissues and mutated in a human mesothelioma. Nature $1993 ; 4: 415-420$.

35) Silver BJ : Platelet-derived growth factor in human malignancy. Biofactors $1992 ; 3$ : 217227.

36) Ke Y, Reddel RR, Gerwin BI, et al : Establishment of a human in vitro mesothelial cell model system for investigating mechanisms of asbestos-induced-meso- thelioma. Am J Pathol 1989 ; 134 : 979-991.

37) Versnel MA, Claesson-Welsh L, Hammacher A, et al : Human malignant mesothelioma cell lines express PDGF b-receptors whereas cultured normal mesothelial cells express predominantly PDGF a-receptors. Oncogene $1991 ; 6: 2005-2011$.

38) Gerwin BI, Lechner JF, Reddel RR, et al : Comparison of production of transforming growth factor-b and platelet-derived browth factor by normal human mesothelial cells and mesothelioma cell lines. Cancer Res $1987 ; 47: 6180-6184$.

39) Pogrebniak HW, Lubensky IA, Pass HI : Differential expression of platelet derived growth factor-b in malignant mesothelioma: A clue to future therapies? Surg Oncol $1993 ; 2: 235-240$.

40) Lee TC, Zhang Y, Aston C, et al : Normal human mesothelial cells and mesothelioma cell lines express insulin-like growth factor I and associated molecules. Cancer Res 1993 ; $53: 2858-2864$.

41) Higashihara M, Sunaga S, Tange T, Oohashi $\mathrm{H}$, Kurokawa $\mathrm{K}$ : Increased secretion of interleukin-6 in malignant mesothelioma cells from a patient with marked thrombocytosis. Cancer $1992 ; 70: 2105-2108$.

42) Schmitter D, Lauber B, Fagg B, Stahel RA : Hematopoietic growth factors secreted by seven human pleural mesothelioma cell lines : Interleukin-6 production as a common feature. Int J Cancer $1992 ; 51: 296-301$.

43) Demetri GD, Zenzie BW, Rheinwald JG, Griffin JD : Expression of colony-stimulating factor genes by normal human mesothelial cells and human malignant mesothelioma cells lines in vitro. Blood $1989 ; 74$ : 940-946.

44) Lauber B, Leuthold M, Schmitter D, CanoSantos J, Waibel R, Stahel RA : An autocrine mitogenic activity produced by a pleural human mesothelioma cell line. Int J Cancer $1992 ; 50: 943-950$.

45) Carbone M, Cicala C, Pompetti F, Festa A, Levine AS : SV40 induces mesotheliomas in 
hamsters. Proc Amer Assoc Cancer Res $1992 ; 33: 393$.

46) Appel JD, Fasy TM, Kohtz DS, et al :
Asbestos fibers mediate transformation of monkey cells by exogenous plasmid DNA. Proc Natl Acad Sci USA 1988 ; 85 : 7670-7674. 